\title{
Spectrum of imaging findings in Klippel- Trenaunay syndrome affecting lower limbs: a report of three cases
}

\author{
Rituparna Das, Ishan Kumar ${ }^{*}$ (D, Ashish Verma and Ram C. Shukla
}

\begin{abstract}
Background: We present a series of three cases of Klippel-Trenaunay syndrome (KTS) presenting with unilateral lower limb involvement demonstrating a wide range of radiological findings.

Case presentation: Case 1: A 61-year-old male presented with varicosities in the right lower limb, which was atrophic with a complete absence of deep venous system in the right limb. Case 2: A 23-year-old woman presented with hypertrophied left lower limb with multiple varicosities showing unusual distribution and intraosseous extension into the distal femur, patella, and tibia. Case 3: A 14-year-old male presented with hypertrophy of the right lower limb overlying macular cutaneous lesions. Capillary malformation was seen underneath the cutaneous lesion and multiple subcutaneous and intramuscular venous malformations were seen. Also noted was persistent embryological vein, i.e., lateral marginal vein of Servelle.

Conclusion: The three cases in our series demonstrate common and unusual findings of KTS and highlight the role of $\mathrm{CT}$ and MR venography in addition to color Doppler sonography to identify the complete extent of the involvement.
\end{abstract}

Keywords: Klipplel Trenaunay syndrome, Imaging, Lower limbs, Deep venous aplasia

\section{Background}

Klippel-Trenaunay syndrome (KTS) is a rare disorder (estimated incidence 3-5/100,000) [1], characterized by a triad of vascular malformation, venous varicosities, and limb hypertrophy. Important differential diagnoses of this condition include Parkes Weber syndrome, Maffucci syndrome, Beckwith-Wiedemann syndrome, and macrodystrophia lipomatosis [2].

Superficial venous malformations of the unilateral lower limbs or varicosities in unusual distribution are the most common findings but deeper extension into the underlying muscle, bone, or even visceral organs like the spleen, liver, pleura, urinary bladder, or colon (submucosal varices) also have been reported [2]. Deep venous abnormalities such as aplasia, duplications, and aneurysmal dilatation have also been identified [2]. Persistence of embryonic veins such as lateral marginal vein of Servelle and sciatic vein is also a feature suggestive of KTS [3].

Although conventional venography is a gold standard procedure, due to its invasive nature, it has been largely replaced by other noninvasive modalities such as color Doppler, CT, or MR venography. Color Doppler sonography is the most commonly employed imaging modality utilized to diagnose KTS. The presence of varicosities in an unusual location (like

\footnotetext{
* Correspondence: ishanjd@gmail.com

Department of Radiodiagnosis and Imaging, Institute of Medical Sciences, Banaras Hindu University, Varanasi 221005, India
} 
lateral aspect of the leg) and deep venous anomalies are highly indicative of Klippel-Trenaunay syndrome. Vascular (venous) malformations present as a network of vascular channels, with interspersed soft tissue component and calcified phleboliths. On spectral Doppler, the low flow vascular malformations demonstrate no flow or monophasic flow. The absence of high flow arteriovenous malformations is characteristic of this syndrome [4]. A plain radiograph of the affected limb may be able to identify phleboliths or intraosseous extension of the malformations. Detailed preoperative mapping of the venous system by a supplementary CT or MR venography may facilitate the identification of the extent of deep-seated malformation and thus can be helpful to prevent worsening of symptoms following intervention on superficial varicosities. Because the majority of the affected patients are children or young adults, the absence of harmful radiation makes MRI more suitable as the principal imaging tool [4]. The advantage of CT venography over MR venography is its significantly lower procedure time and higher spatial resolution.

The management of KTS is mainly conservative [5-7] and usually, patients have to be on lifelong follow-up. Treatment is mainly aimed at providing symptomatic relief, management of any bleeding episode, and prevention and treatment of complications such as deep vein thrombosis, cellulitis, chronic coagulopathy, and congestive heart failure.

\section{Case presentation \\ Case 1}

A 61-year-old male patient presented with nonhealing ulcer in the right foot with varicosities in the right lower limb, which was present since childhood. He was being managed conservatively for the varices. Physical examination revealed varicose veins in the right thigh and leg and there was overall atrophy of the involved limb (Fig. 1). The patient had undergone a radiograph of the right lower limb which showed shortening of the right femur with osteonecrosis of the femoral head, shallow dysplastic acetabula, and coxa vara deformity, which concurred with the previous history of hip tuberculosis elicited by the patient (Fig. 2a). Surgical treatment of the varicose vein was planned and the patient was referred for a preoperative color Doppler evaluation study of varicose veins. Doppler study revealed extensive varicosities involving the right great saphenous vein and dilated lesser saphenous veins showing venous incompetance. No intersaphenous anastomoses were identified. Scanning of the deep venous system showed a complete

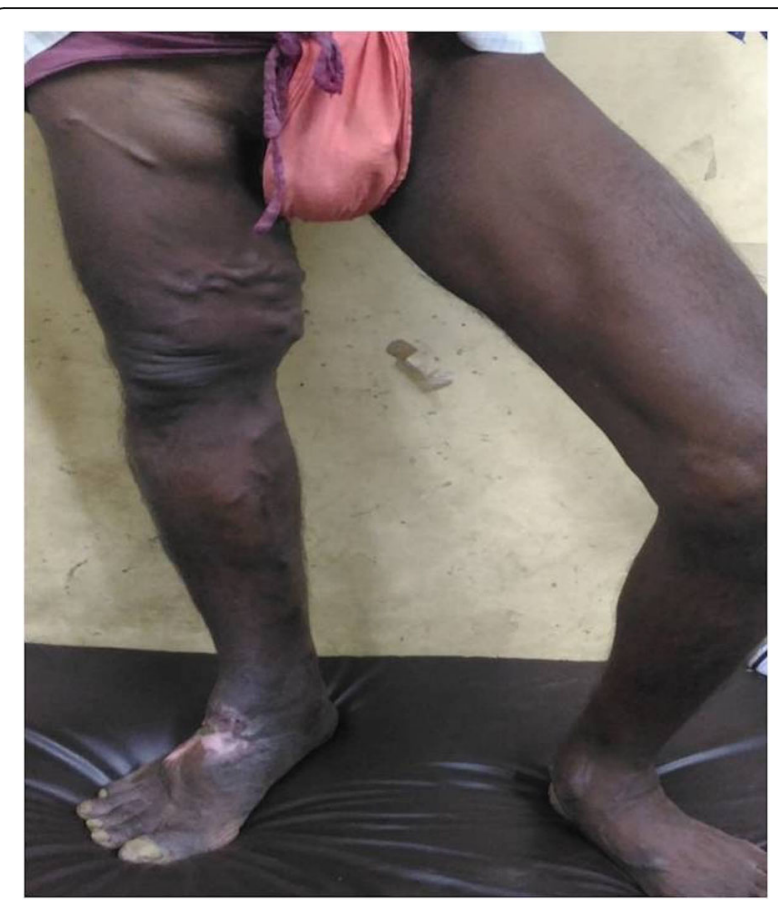

Fig. 1 Clinical photograph of first patient showing varices in right thigh and right leg with a healing venous ulcer in the dorsum of the right foot. There is shortening of the right lower limb as compared to the left lower limb

absence of right external iliac, common and superficial femoral, profunda femoris and popliteal veins. Suspecting a congenital agenesis of deep venous system, a CT venography was performed using a multidetector row 64-slice CT scanner (light speed, GE medical systems, Milwaukee, WI, USA) which confirmed the Doppler findings of complete absence of deep venous system of the right lower limb in addition to atrophic right lower limb, extensive varicosities, (Fig. 2). The patient was managed conservatively as the absence of the deep venous system precluded any active surgical or radiological intervention to ablate the superficial venous varicosities.

\section{Case 2}

A 23-year-old woman presented with hypertrophied left lower limb with multiple varicosities in the affected limb present since childhood. On physical examination, there was left lower limb hypertrophy with prominent tortuous vascular channels along the medial and lateral aspect of the lower limb. No evidence of any skin discoloration or ulceration was noted. Plain radiography showed osseous and soft tissue hypertrophy of the left lower limb. Diffuse cortical thickening of the femur, 


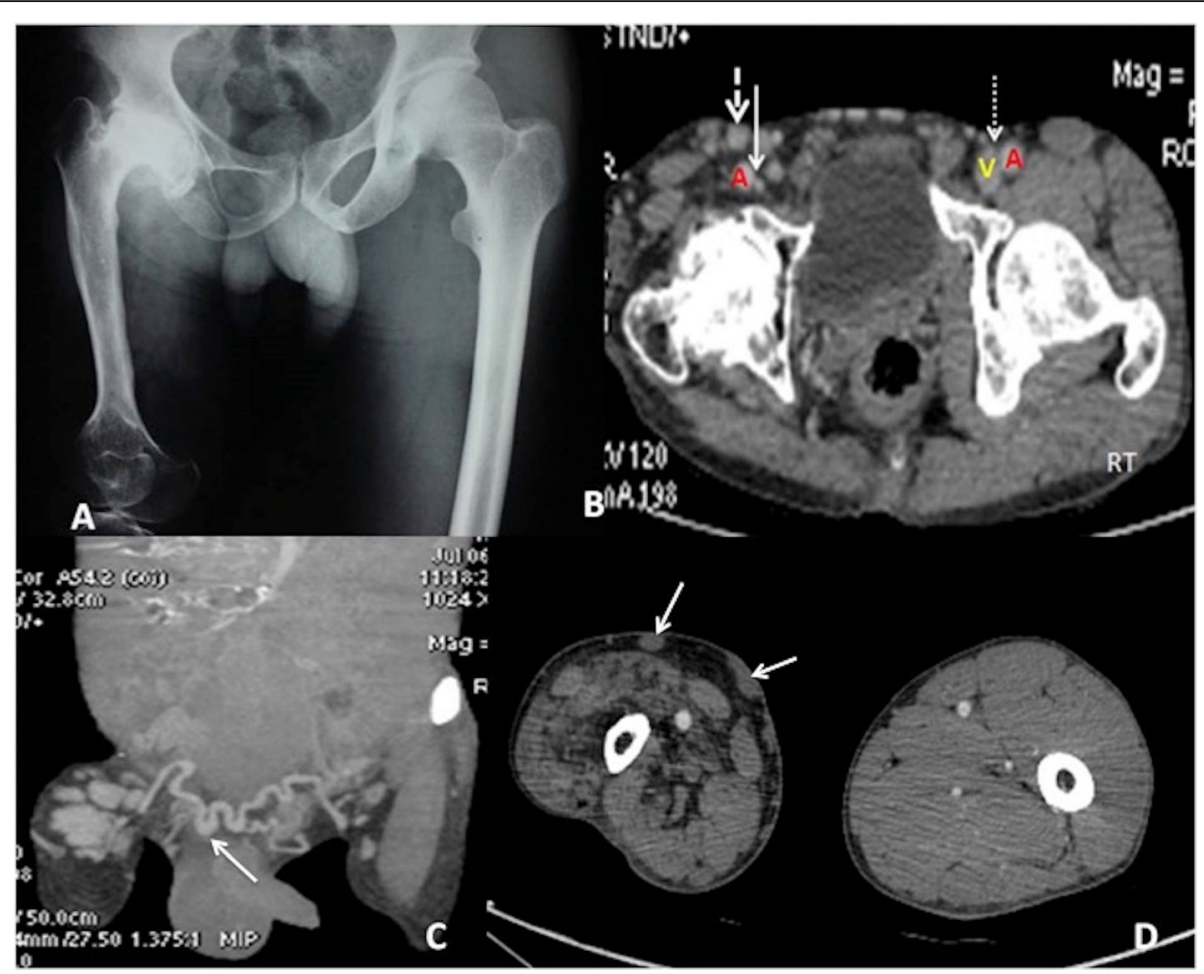

Fig. 2 a X-ray of pelvis including bilateral hip joints and proximal femurs show shallow dysplastic right acetabulum, osteonecrosis of the femoral head, decreased hip joint space, coxa vara deformity, and increased cortical thickness of the short right femur. b Axial contrast enhanced $C T$ angiography image demonstrates superficial varicosities (interrupted arrow) and absent external iliac vein on right side (arrow). Normal relationship of artery (A) and vein $(V)$ on left side with presence of external iliac vein (dotted arrow). c Coronal contrast enhanced CT venography image (delayed phase) showing venous arch in anterior abdominal wall (arrow) connecting venous system of both lower limbs. d Axial contrast enhanced $C T$ angiography image demonstrates muscular atrophy of the right thigh and multiple subcutaneous varicosities (arrow)

tibia, and fibula was seen. No bony deformity was observed. The patient was referred for color Doppler evaluation which revealed multiple superficial and intramuscular varicosities involving the left thigh and leg. The varicosities showed both usual (medial aspect) and unusual (lateral) distribution. Both the saphenofemoral and saphenopopliteal junctions were incompetent and showed reflux times of $2-3 \mathrm{~s}$ and 4$6 \mathrm{~s}$, respectively. The deep venous system did not show any evidence of thrombosis or reflux. Multiple dilated perforator vessels were identified in the thigh and calf. No intersaphenous anastmoses were seen. A suspicion of KTS was raised on color Doppler based on the unusual distribution of the varicosities and intramuscular extension, and $\mathrm{CT}$ venography was performed.

CT angiography revealed multiple varicosities along the superficial venous system and abnormal vascular channels in intramuscular and superficial planes showing contrast filling on delayed scans suggestive of venous malformations (Fig. 3). In addition, the malformed vessels were seen showing an intraosseous extension into distal femur, patella, and tibia. Also, there was evidence of soft tissue, muscular and osseous hypertrophy of the left lower limb. The patient was managed by US-guided sclerotherapy of abnormal vascular channels and is on follow-up.

\section{Case 3}

A 14-year-old male presented with hypertrophy of the right lower limb and multiple venous prominences in the lateral aspect of the limb and overlying cutaneous lesions. Physical examination revealed an increased girth and length of the right lower limb and multiple varicosities in the anterolateral aspect of the right thigh and leg. Multiple macular lesions were noted along the anterior and lateral aspects of the right leg (Fig. 4). Color Doppler revealed multiple superficial and intramuscular varicosities in the right thigh and leg in the anterior, posterior, and lateral compartments. An 


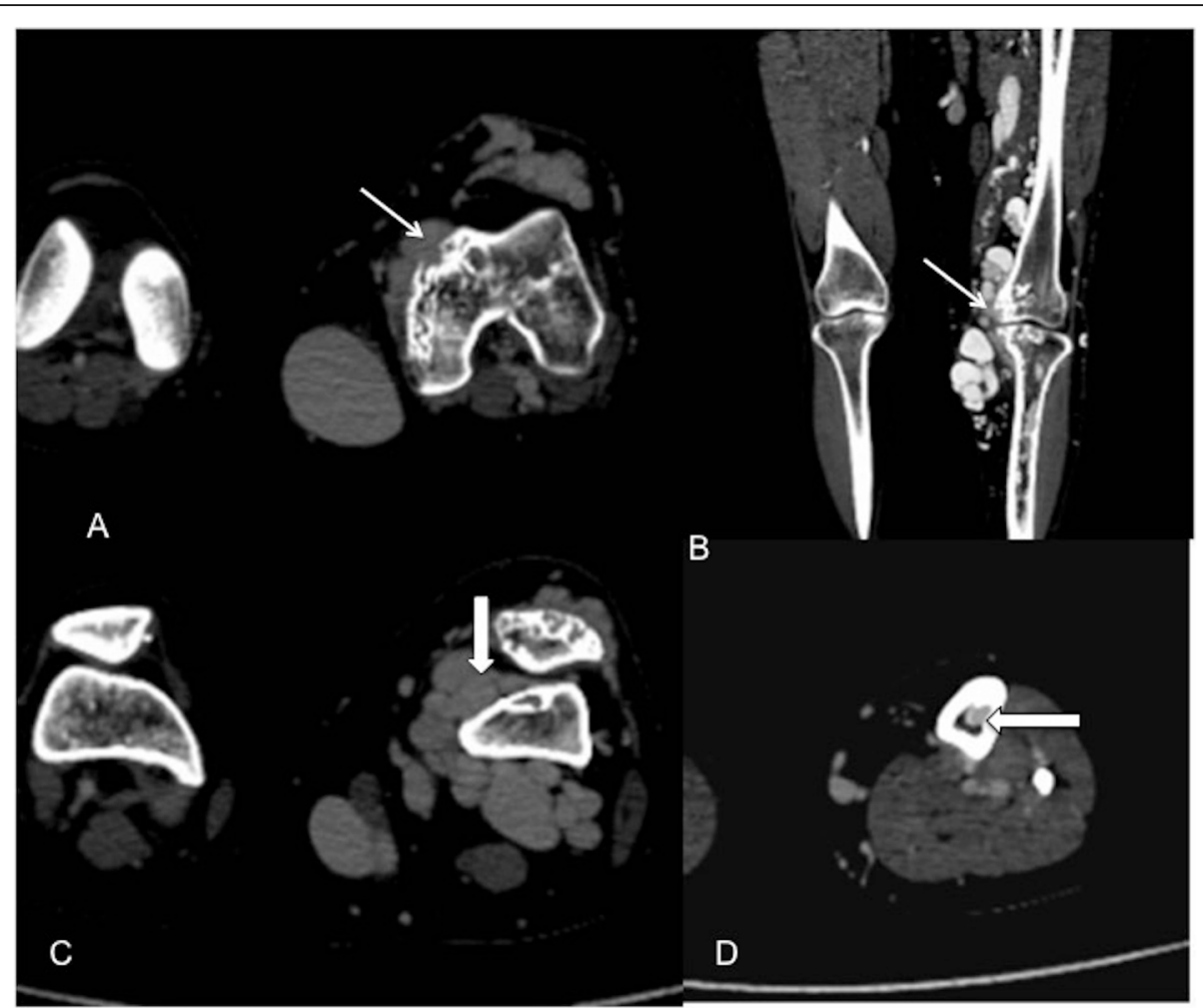

Fig. 3 a-d Axial and coronal contrast-enhanced CT images showing hypertrophy of subcutaneous tissue of the left thigh with multiple dilated venous channels in subcutaneous and intermuscular planes (predominantly in anteromedial distribution) with extension into patellofemoral joint space (c; solid arrow). These varicous channels are seen causing cortical bony erosions ( $\mathbf{a}$, $\mathbf{b}$; arrows) as well as frank intraosseous extension (d, solid arrow)

abnormal lateral marginal vein and multiple dilated perforator channels were identified in the calf and thigh with evidence of venous reflux. The saphenofemoral junction was competent while saphenopopliteal junctions showed Doppler evidence of venous insufficiency with reflux time of $3 \mathrm{~s}$. The deep venous system did not show any evidence of thrombosis or venous insufficiency. No intersaphenous anastomotic channels were identified. Underlying the cutaneous macular lesions, ill-defined subcutaneous lesions having a tuft of small vascular channels and minimal soft tissue components were seen. The vessels showed slow flow on spectral Doppler study, and the lesions were identified as capillary malformations. On CT angiography, there was evidence of multiple dilated vascular channels in subcutaneous and intramuscular plane (involving hamstrings and peroneal muscles) which showed early filling in the arterial phase with slow washout in delayed phase images (Fig. 5). Multiple subcutaneous soft tissue density lesions with surrounding dilated vessels were seen in areas corresponding to the cutaneous malformations in the patient. There was osseous and soft tissue hypertrophy of the right lower limb. MRI was performed to look for any other vascular malformation and assess soft tissue extension of the varicosities (Fig. 5). MRI confirmed the CT findings and also demonstrated the lateral marginal vein of Servelle (Fig. 5d) running along the lateral aspect of the leg.

The patient has been planned for endovenous laser ablation of the superficial varicosities. For compensating the limb length discrepancy, the patient was advised to use customized shoes. He has been counseled regarding the use of stockings and avoiding any risk factor for recurrence of varices.

\section{Discussion}

KTS is a syndrome characterized by extremely variable clinical features and severity of symptoms as well as remarkable variability in the expression of its components. Over the years, various authors have utilized different diagnostic criteria for this entity. Moreover, with the evolution of advanced imaging techniques 


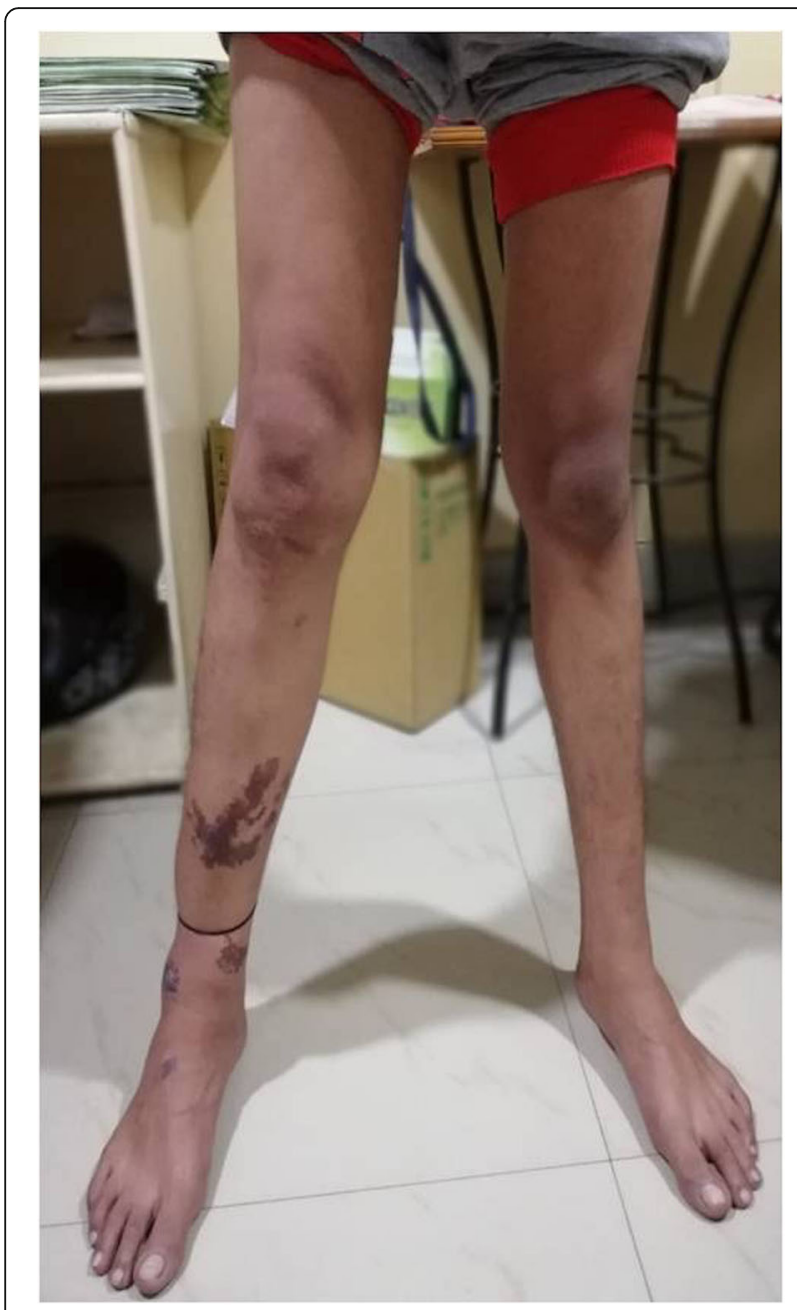

Fig. 4 Clinical photograph of the patient (case 3) showing hypertrophied right lower limb with multiple cutaneous macular lesions in the right leg and increased ability to detect and characterize the various vascular malformations, the diagnostic criteria have also evolved. A review by Oduber et al. has proposed a strict diagnostic criterion while respecting the variable nature of the disease [8]. According to this study, KTS is characterized by two major essential diagnostic features, i.e. (a) congenital vascular malformation and (b) growth disturbance of the limb. Vascular malformations in KTS have further been classified into (a) capillary malformation (port-wine stain) (b) venous malformation (c) arteriovenous malformation, and (d) lymphatic malformations, of which, the presence of the first two is essential for the diagnosis of KTS. A venous malformation in this classification has been applied as a blanket term used to encompass varicose veins, hypoplasia or aplasia of veins, and persistence of fetal veins. Growth disturbance in KTS has been defined as hypertrophy or hypotrophy of a small or large body parts ranging from involvement of isolated finger to involvement of the entire limb or half of the entire body. According to this definition, all three patients in our study satisfied the criteria to be labeled as KTS. Hypotrophy/atrophy, as present in our first case, is an extremely uncommon feature of KTS, with only a few reports [9]. Other imaging findings that are encountered in KTS, but do not contribute to the essential diagnostic criteria, are autonomic dysfunction, presence of cavernoma, hemangioma, aneurysm, hemimegalencephaly as well as gastrointestinal and genitourinary vascular anomalies, polydactyly/syndactyly, and positional limb defects such as talipes and metatarsus varus $[8,10]$..

The evaluation of the severity of KTS is most commonly centered at the assessment of clinical severity of venous abnormality which included extent and distribution of structural and functional venous anomaly. Comprehensive assessment of the involved limb using Doppler sonography can provide a measure of severity of venous insufficiency in the superficial venous system. In addition, the evaluation of perforator system and deep venous system should be to classify the extent of venous reflux into triple (superficial, deep, and perforator) or dual (superficial with either deep or perforator) venous insufficiency. In addition, the presence of deep venous hypoplasia, deep venous thrombosis, limb edema, leg ulcers, superficial thrombophlebitis, intraosseous extension of vascular malformation, arthritis, and neuropathy in the affected limb contributes to the morbidity. Although we did not perform a quantitative pulsed-wave Doppler evaluation of venous insufficiency in our cases, the absence of deep venous system in case 1 and the presence of an intraosseous extension in case 2 pointed towards higher disease severity [11].

\section{Conclusion}

Our series revealed variable imaging findings of KTS afflicting lower limbs. A high degree of suspicion is required for diagnosis of KTS and suspicion should be raised in the presence of few salient imaging findings such as history since birth, unilateral limb hypertrophy, and atypical distribution of varices, which may show intramuscular and intraosseous extension. One of our patients presented with atrophy that might be attributed to its late presentation in his 60s. The imaging evaluation of the superficial venous system should be aimed at recognizing the unusual distribution of varicosities of low-flow venous malformations and identification of persistent embryological veins such as lateral marginal vein. Deep venous system 


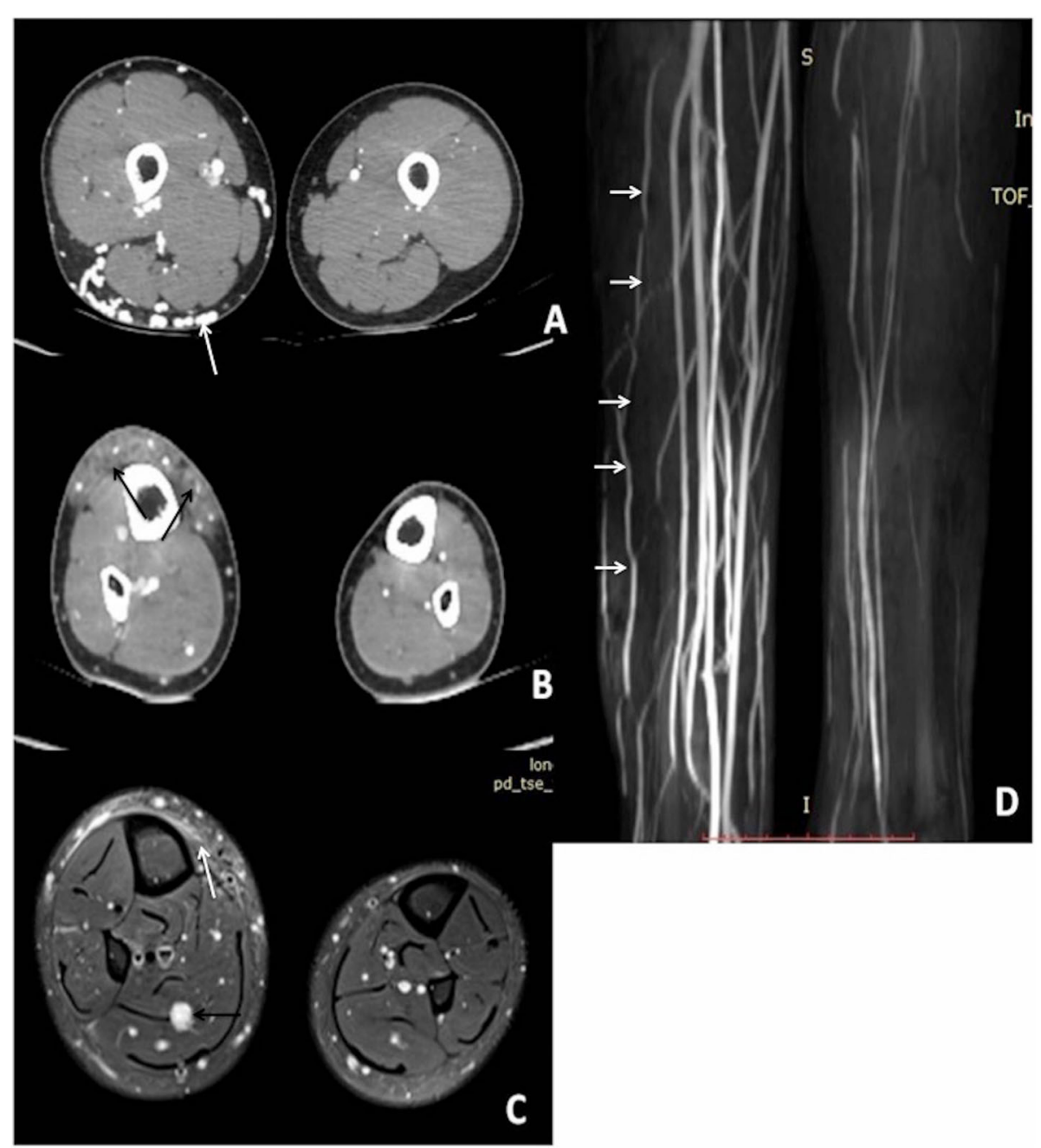

Fig. 5 Axial contrast-enhanced CT showing hypertrophied right thigh with multiple subcutaneous varices (white arrow in a) and well defined subcutaneous capillary malformation in anterior aspect of right leg (black arrow in b). Axial proton density MR image (c) showing hypertrophied right leg with subcutaneous capillary malformations (white arrow in c) and intramuscular varices (black arrow in c). MIP TOF 2D MR venogram image (d) showing lateral marginal vein of Servelle (red arrow), multiple varicosities, and normal deep venous system of the right lower limb

should be carefully evaluated for partial or complete agenesis or duplication. Our series also demonstrates the utility of obtaining a supplementary CT or, preferably, MR venography to evaluate the complete extent of the disease which may affect the underlying bones or viscera.

\section{Abbreviations}

CT: Computed tomography; KTS: Klippel-Trenaunay syndrome; MRI: Magnetic resonance imaging

\section{Acknowledgements}

None

\section{Authors' contributions}

$\mathrm{RD}$ and IK contributed to the work up and draft of the manuscript. AV and RCS contributed to the proof reading and finalization of the manuscript. All authors have read and approved the manuscript.

\section{Funding}

This study had no funding from any resource.

\section{Availability of data and materials}

The datasets used and/or analyzed during the current study are available from the corresponding author on reasonable request.

\section{Ethics approval and consent to participate}

This was a report of three cases and the Institute Review Board (IRB) of Institute of Medical sciences - Banaras Hindu University does permit the publication of case reports/series which are not categorized as "original research," or "a systematic investigation." The case reports/series can be published with the consent of the patient/legal guardian.

\section{Consent for publication}

Written informed consent has been taken from the adult patients in the study for publication. Informed consent has been obtained from the parent of the minor patient in this case series.

\section{Competing interests}

The authors declare that they have no competing interests. 
Received: 30 August 2019 Accepted: 22 December 2019

Published online: 30 December 2019

\section{References}

1. Jacob AG, Driscoll DJ, Shaughnessy WJ, Stanson AW, Clay RP, Gloviczki P (1998) Klippel Trenanuay syndrome: Spectrum and management. Mayo Clin Proc 38:36-37

2. Cha SH, Romeo MA, Neutze JA (2005) Visceral manifestations of Klippel-Trenaunay syndrome. Radiographics 25:1694-1697

3. Rojas Martinez R, Puech-Leão P, Guimarães PM et al (2001) Persistence of the embryonic lateral marginal vein: report of two cases. Rev Hosp Clin Fac Med Sao Paulo 56:159-162

4. Nozaki T, Nosaka S, Miyazaki O, Makidono A, Yamamoto A, Niwa T, Tsutsumi Y, Aida N, Masaki H, Saida Y (2013) Syndromes associated with vascular tumors and malformations: a pictorial review. RadioGraphics 33(1):175-195

5. Reddy OJ, Gafoor JA, Rajanikanth M, Prasad PO (2015) Klippel-Trenaunay syndrome with review of literature. J NTR Univ Health Sci 4:120-123

6. Wilson $\mathrm{CL}$, Song LM, Chua $\mathrm{H}$ et al (2001) Bleeding from cavernous angiomatosis of the rectum in Klippel-Trenaunay syndrome: report of three cases and literature review. Am J Gastroenterol 96:2783-2788

7. Ernemann U, Kramer U, Miller S et al (2010) Current concepts in the classification, diagnosis and treatment of vascular anomalies. Eur J Radiol 75(1):2-11

8. Oduber CE, van der Horst CM, Hennekam RC (2008) Klippel-Trenaunay syndrome: diagnostic criteria and hypothesis on etiology. Ann Plast Surg 60: 217-222

9. Freund E (1939) Diffuse genuine phlebectasia. Arch Surg 60:113-121

10. Abdel Razek AAK (2019) Imaging findings of Klippel-Trenaunay syndrome. J Comput Assist Tomogr 43:786-792

11. Delis KT, Gloviczki P, Wennberg PW, Rooke TW, Driscoll DJ (2007) Hemodynamic impairment, venous segmental disease, and clinical severity scoring in limbs with Klippel-Trenaunay syndrome. J Vasc Surg 45(3):561-567

\section{Publisher's Note}

Springer Nature remains neutral with regard to jurisdictional claims in published maps and institutional affiliations.

\section{Submit your manuscript to a SpringerOpen ${ }^{\circ}$ journal and benefit from:}

- Convenient online submission

- Rigorous peer review

- Open access: articles freely available online

High visibility within the field

- Retaining the copyright to your article

Submit your next manuscript at $\boldsymbol{\nabla}$ springeropen.com 